\title{
Robot-Fish Interaction Helps to Trigger Social Buffering in Neon Tetras: The Potential Role of Social Robotics in Treating Anxiety
}

\author{
Donato Romano ${ }^{1,2}$ (1) Cesare Stefanini ${ }^{1,2,3}$
}

Accepted: 30 August 2021 / Published online: 17 December 2021

(c) The Author(s) 2021

\begin{abstract}
The emerging field of social robotics comprises several multidisciplinary applications. Anxiety and stress therapies can greatly benefit by socio-emotional support provided by robots, although the intervention of social robots as effective treatment needs to be fully understood. Herein, Paracheirodon innesi, a social fish species, was used to interact with a robotic fish to understand intrinsic and extrinsic mechanisms causing anxiety, and how social robots can be effectively used as anxiety treatments. In the first experiment we tested the effects of a conspecific-mimicking robot on the fish tendency to swim in the bottom when transferred in a new tank. Here, $P$. innesi spent a significantly longer time in the upper section of the test tank when the robotic fish was present, clearly indicating a reduction of their state of anxiety due to social stimuli. The second experiment was based on a modification of the dark/light preference test, since many teleost fish are scototactic, preferring dark environments. However, when the robotic fish was placed in the white half of the test tank, $P$. innesi individuals swam longer in this section otherwise aversive. Social support provided by the robotic fish in both experiments produced a better recovery from anxiety due to social buffering, a phenomenon regulated by specific neural mechanisms. This study provides new insights on the evolution and mechanisms of social buffering to reduce anxiety, as well as on the use of social robots as an alternative to traditional approaches in treating anxiety symptoms.
\end{abstract}

Keywords Animal-robot interaction · Anxiety $\cdot$ Biohybrid system $\cdot$ Ethorobotics $\cdot$ Social buffering $\cdot$ Social robotics

\section{Introduction}

Social robotics is an emerging multidisciplinary field whose aim is to develop life-like robotic systems socially accepted by humans [1-3]. Social robots applications comprise several domains including education, therapy, assistive robotics, human-robot coordinated tasks, search and rescue, domestic aspects $[4,5]$. Since people tend to anthropomorphize social robots, biomimetic design principles are of crucial importance in this field to ensure robots' interaction and acceptance $[6,7]$.

Donato Romano

donato.romano@santannapisa.it

1 The BioRobotics Institute, Sant' Anna School of Advanced Studies, Viale Rinaldo Piaggio 34, 56025 Pontedera, Pisa, Italy

2 Department of Excellence in Robotics and AI, Sant'Anna, School of Advanced Studies, 56127 Pisa, Italy

3 Healthcare Engineering Innovation Center (HEIC), Khalifa University, Abu Dhabi, UAE
The wide spectrum of application of social robots also includes their use in reducing anxiety and stress effects to improve the quality of life of patients [8,9]. Anxiety disorders produce strong suffering and dysfunctions in 5-20\% of children [10,11] and in $18 \%$ of adults [12] and include specific or social phobias, panic, post-traumatic dysfunctions, obsessive-compulsive disorders [13]. The use of social robots to reduce anxiety by providing socio-emotional support, has been found to play an important role in different contexts, and especially during paediatric health-care $[14,15]$.

Robotic solutions aimed at designing human-centered systems are often inspired from nature. Since animals are important model organisms to study factors contributing to anxiety [16-20], they can be used to investigate and refine the intervention of social robots as effective treatment. Furthermore, social robotics techniques can be also used to improve the welfare and health-care of domestic animal species [21]. Indeed, animal health-care, animal welfare, food safety and public health have become of primary importance in policy, as pointed out by the European Food Safety Authority (EFSA) [22, 23]. 
The genus Paracheirodon, along with some other teleost fish genera, represents an emergent biological model to investigate anxiety-like reactions [19, 24-26]. In this context, we used the neon tetra Paracheirodon innesi, Myers (Characiformes: Characidae), a common and easy to maintain ornamental fish showing robust social behaviour [27, 28].

The use of robotic fish mimicking conspecifics of $P$. innesi can be useful to understand which intrinsic and extrinsic mechanisms cause anxiety, as well as how social robots can be effectively used as pathological anxiety treatments. Animal-robot interactions and ethorobotics are advanced biorobotic and bionic paradigms merging robotics with ethology that enable to establish biohybrid social systems useful for multidisciplinary purposes [29-37].

Herein, we developed a robotic platform actuating a robotic fish inspired by $P$. innesi colour and morphology to investigate the biological mechanisms of social behaviours to effectively use robotic social stimuli in reducing anxiety and posttraumatic stress responses.

Anxiety behaviours in $P$. innesi was assessed by conducting experiments based on the novel tank diving test, a well-established method to assay for anxiety as a consequence of stressful manipulations in fish [38]. When in a new environment, fish tend to spend a significantly longer time in the bottom of the tank. We measured the duration in the bottom of the tank in both $P$. innesi exposed to the robotic fish, and non-exposed to the robotic fish to quantify beneficial effects of social stimuli.

To further understand the impact of social biomimetic robots in ameliorating anxiety symptoms, we carried out additional experiments based on a modification of the dark/light preference test, an etho-experimental anxiety model measuring locomotor activity of fish in both dark and light environments as an anxiety index [24]. Several genera of teleosts, including the genus Paracheirodon, were found scototactic (e.g. the preferential pattern of exploration for dark environments) [24, 39]. Here, we presented a conflict situation by locating the robotic fish in the light environment, naturally aversive for these fish, and measuring the time spent by neon tetras in both environments.

The animal-robot interaction approach we propose would represent an elective strategy to integrate the usefulness of animals as models in anxiety-related studies, with investigation on the role of social robots as possible therapy.

\section{Materials and Methods}

\subsection{Ethics Statement}

The present study adheres to the Guidelines for the Use of Animals in Research [40], and to the 7010-2020_-IEEE Rec- ommended Practice for Assessing the Impact of Autonomous and Intelligent Systems on Human Well-Being [41], as well as to the legal requirements of Italian legislation (D.M. 116,192), and EU regulation [42]. All experiments consisted in behavioural tests, and no specific permits are needed in the country where the experiments were conducted.

\subsection{Animals Rearing and General Observations}

Adult fish of the species Paracheirodon innesi were purchased from a pet store in Pontedera (Pisa, Italy). Fish were maintained under laboratory conditions (at $25 \pm 1{ }^{\circ} \mathrm{C}$, and 16:8 h light:dark photoperiod) in $100 \mathrm{~L}$ aquaria that were filled with activated charcoal-filtered water, at The BioRobotics Institute of Sant'Anna School of Advanced Studies (Pisa, Italy). Cultures were constantly aerated by an air diffuser, and the $30 \%$ of the water was replaced every third day. Fish diet consisted in a commercial food (Tetramin ${ }^{\circledR}$ flake food) that was provided twice a day ad libitum. The aforementioned controlled conditions were the same also during experiments. The laboratory was illuminated by overhead fluorescent daylight tubes (Philips 30 W/33). Diffused laboratory lighting were used to reduce reflection and phototaxis. In each experiment, the behaviour of $P$. innesi was directly recorded by an observer. Test tanks and the robotic fish were accurately washed at the end of each replicate [32], to avoid effects due to olfactory cues from previous tests. All $P$. innesi individuals were tested only once.

\subsection{Robotic Fish and Experimental Apparatus}

The robotic fish was designed to resemble $P$. innesi as much as possible (Fig. 1a), and included a dorsal fin, a second dorsal fin, an anal fin, a caudal fin, two pelvic fins, and two ocular areas. Four parts for each sagittal section of the fish were designed in SolidWorks (Dassault Systemes, VelizyVillacoublay, France), and fast prototyped in acrylonitrile butadiene styrene (ABS). The final eight parts were assembled by placing a chiffon fabric rectangle $(18 \times 3 \mathrm{~mm})$ as fish's sagittal plane, between complementary parts (Fig. 1b). The robotic fish was $4 \mathrm{~mm}$ wide, $11 \mathrm{~mm}$ tall, and $27 \mathrm{~mm}$ long. To mimic the color pattern of $P$. innesi the robotic fish was painted with non-toxic pigments (Fig. 1c). A standard CIELab colour space coordinates determined using a colorimeter (Nix Pro 2 Color Sensor) was used to record colour measurements of the $P$. innesi body, and of the robotic fish body. A thin layer of transparent silicone rubber (Dragon Skin) covered the robotic fish (Fig. 1d). This fabrication process enabled to have a finalized robotic fish with an increased biomimetic appearance, also allowing passive body undulations, due to its soft and compliant body (Fig. 1c).

The robotic fish was hinged to a magnetic base by a rod. The magnetic base was magnetic coupled with a trajectory 

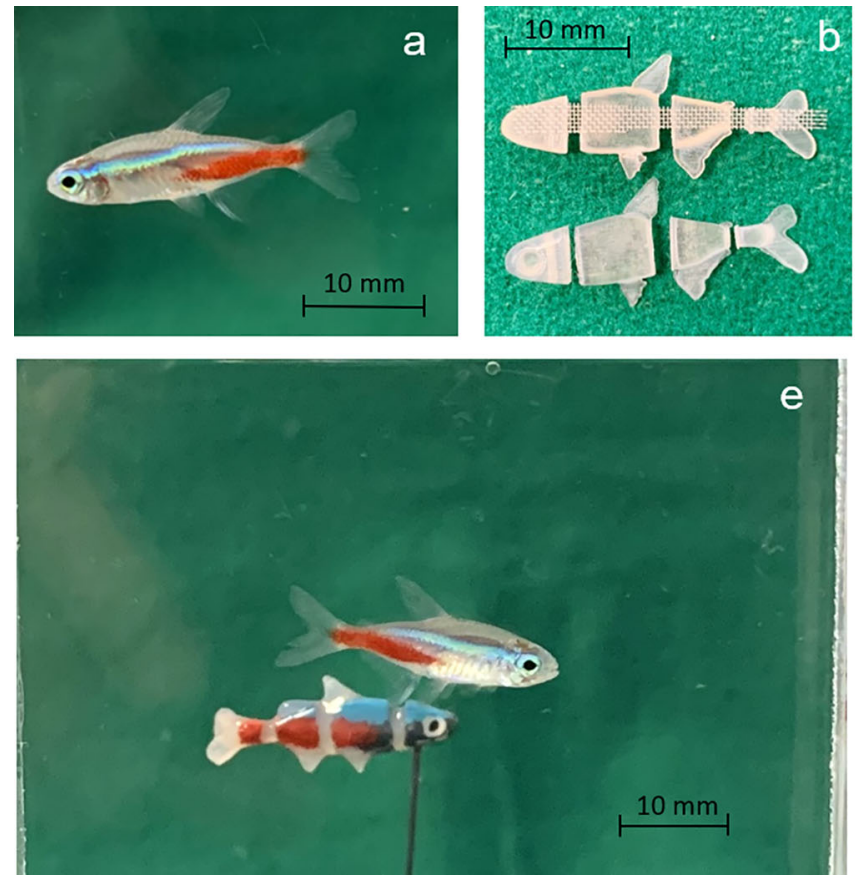
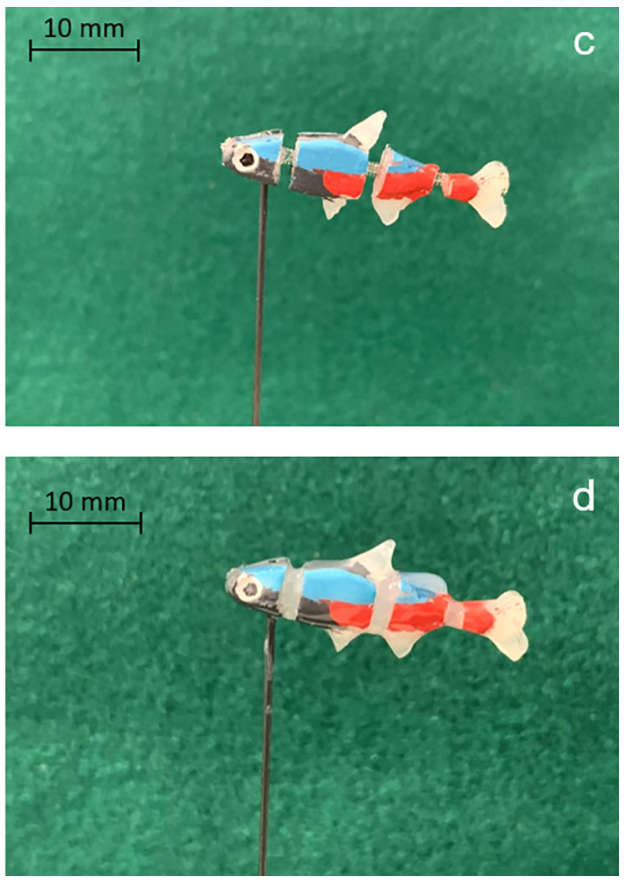

Fig. 1 Development phases of the robotic fish. The neon tetra Paracheirodon innesi (a) was used as model to design the conspecificmimicking robotic fish. The robotic fish consisted of 4 parts for each sagittal section that were assembled by placing a chiffon fabric rectangle between complementary parts (b). The robotic fish was then painted with non-toxic pigments to mimic the color pattern of $P$. innesi (c), and covered by a thin layer of transparent silicone rubber to increase its biomimetic soft appearance (d). A snapshot from the animal-robot social interaction showing a $P$. innesi individual swimming close to the finalized robotic fish (e) generator below the test tank that moved directly the robotic fish. The trajectory generator had an operating area of around $400 \times 200 \mathrm{~mm}$ (path following accuracy $=0.01 \mathrm{~mm}$ ), and included two stepper motors, actuating two sliding axis (i.e., $x$ and $y$ axes), and a microcontroller (Arduino Nano) [35]. Plotted trajectories were sent to the microcontroller after conversion in G-Code (i.e., RS-274). An external processor connected to the microcontroller, was used to manage the plotting and code conversion phases.

\subsection{Experiment 1: Effect of a Social Robot on the Explorative Behaviour of a Novel Tank}

Fish were individually placed in a test tank $(400 \times 300 \times$ $150 \mathrm{~mm}$; length $\mathrm{x}$ width $\mathrm{x}$ depth) whose water column was divided in two virtual sections: upper section, bottom section (Fig. 2a).

Since the novel tank diving test is based on evoking anxiety behaviour in fish when in a new environment [38], no acclimatation periods was applied from the transfer of fish in the test tank and the start of the test.

Fish were exposed to 2 treatments: (i) robotic social stimuli; (ii) no social stimuli. In the treatment i) the robotic fish was located $30 \mathrm{~mm}$ below the surface and moved on an ellip- tical trajectory (semi-major axis $195 \mathrm{~mm}$; semi-minor axis $80 \mathrm{~mm}$ ), with a velocity of $5 \mathrm{~mm} / \mathrm{s}$.

For each treatment, the time spent by $P$. innesi in each section of the test tank was measured, considering as index of anxiety the choice of position in the bottom vs. upper sections [38]. The time needed to start swimming (latency) after the release in test tank was also recorded. The test lasted $15 \mathrm{~min}$. Twenty fish were analysed. Each fish was tested only once.

\subsection{Experiment 2: Effect of a Social Robot on a Dark/Light Preference Context}

Herein, a test tank $(400 \times 300 \times 150 \mathrm{~mm}$; length $\mathrm{x}$ width $\mathrm{x}$ depth) with half black/half white walls and bottom coloured was used to analyses the effect of the robotic fish on the dark/light preference of fish [19] (Fig. 2b). The test tank included a central Sect. $(133 \times 300 \times 150 \mathrm{~mm})$ bounded by removable partitions coloured with the same colour of the test tank side.

$P$. innesi individual were transferred in the central section for five minutes for acclimatation. Once partitions were removed, the test started and lasted $15 \mathrm{~min}$. 
Fig. 2 Schematic representations depicting the experiment 1 (a), and the experiment 2 (b)
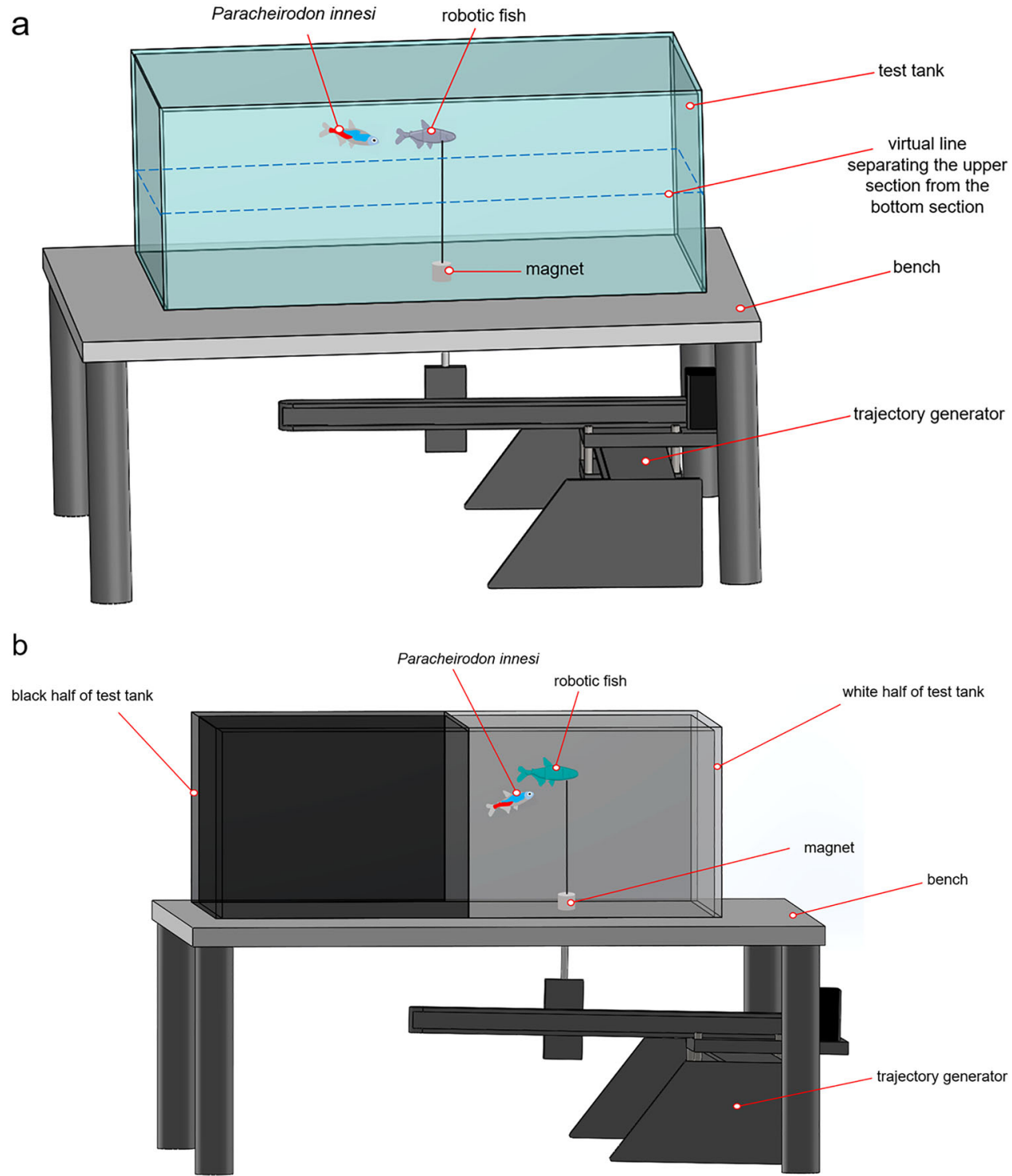

Three treatments were proposed: (i) robotic fish in the white half; (ii) robotic fish in the black half; (iii) no robotic fish.

When the robotic fish was presented in one of the two halves, it moved on a circular trajectory $(\varnothing 100 \mathrm{~mm})$, with a velocity of $5 \mathrm{~mm} / \mathrm{s}$. For each treatment, we measured the time spent by $P$. innesi in the black and in the white halves of the test tank, as well as the time needed for the first choice of half. We also focally measured the schooling behaviour of $P$. innesi individuals towards the robotic fish, defined as the tendency of fish to swim at a distance of at least 5 body lengths from other conspecifics and moving collectively [43]. However, Seghers and Magurran [43] also reported that generally fish are observed to school closer than the afore-mentioned distance, and this was also confirmed by our observations.
After each trial the test tank was rotated to avoid orientation effects. Twenty fish were analysed, and only a single test was carried out for each subject.

\subsection{Statistical Analysis}

Data on the impact of the time spent in different sections of the test tank, the duration of the latency, as well as differences among $P$. innesi fish exposed to robotic social stimuli and no social stimuli were analysed by using non-parametric statistics (Wilcoxon test, $P=0.05$ ), as they were not normally distributed (Shapiro-Wilk test, goodness of fit $P<0.05$ ). Differences in the time spent by $P$. innesi in the black and in the white halves of the test tank, the time needed for the first choice, as well as the schooling behaviour duration, postexposure to the robotic fish in the white half, the robotic fish in the black half, and no robotic fish, were also not normally 
distributed. Therefore, we relied on Kruskal-Wallis test followed by Steel-Dwass test $(P=0.05)$ to analyse them. All data were analysed by R software v3.6.1 (Stats Package).

\section{Results}

\subsection{Experiment 1: Effect of a Social Robot on the Explorative Behaviour of a Novel Tank}

Robotic social stimuli significantly affected the time fish spent in different sections of the test tanks, and in particular $P$. innesi individuals spent more time in the upper section when the robotic fish was present compared to when no stimuli were provided $(Z=5.032 ; P<0.0001)$ (Fig. 3a). When no social stimuli were presented $P$. innesi individuals spent more time in the bottom section $(Z=-4.964 ; P<0.0001)$ (Fig. 3b).

The time spent in different sections was importantly influenced by social stimuli.

The time spent in the bottom section lasted significantly more when no social stimuli were presented $(Z=-4.966 ; P$ $<0.0001$ ) (Fig. 3c). The time spent in the upper section lasted significantly more when the robotic fish was exposed, $(Z=$ 5.033; $P<0.0001)$ (Fig. 3d). The latency duration decreased significantly when the robotic fish was presented $(Z=-$ 4.126; $P<0.0001$ ) (Fig. 3e).

\subsection{Experiment 2: effect of a social robot on a dark/light preference context}

The presence and location of the robotic fish in the test tank significantly affected the time spent by fish in the black half $\left(\chi^{2}=40.928 ;\right.$ d.f. $\left.=2 ; P<0.0001\right)$, and in the white half $\left(\chi^{2}\right.$ $=41.089 ; d . f .=2 ; P<0.0001)$. The time spent in the black half of the test tank was longer when the robotic fish was in the black half compared to when the robotic fish was in in the white half $(Z=-5.396 ; P<0.0001)$. The time spent in the black half of the test tank was longer when the robotic fish was not present compared to when the robotic fish was in the white half $(\mathrm{Z}=-5.399 ; P<0.0001)$ (Fig. 4a).

Fish spent more time in the white half of the test tank when the robotic fish was in the white half compared to when the robotic fish was in in the black half $(Z=-5.396 ; P$ $<0.0001)$. Fish spent more time in the white half of the test tank when the robotic fish was in the white half compared to when the robotic fish was not present $(Z=-5.399 ; P$ $<0.0001$ ) (Fig. 4b).

The time needed by fish for the first choice of half of the test tank was significantly affected by the presence and location of the robotic fish in the test tank $\left(\chi^{2}=36.864 ;\right.$ d.f.
$=2 ; P<0.0001)$. The time needed for the first choice was longer when the robotic fish was not present compared to when the robotic fish was in the black half $(Z=-5.184$; $P$ $<0.0001)$, and when the robotic fish was in the white half $(Z$ $=-5.021 ; P<0.0001$ ) (Fig. 4c).

The duration of schooling behaviours was not significantly affected by the location of the robotic fish in the test tank $\left(\chi^{2}\right.$ $=2.681 ;$ d.f. $=1 ; P=0.101$ ) (Fig. $4 \mathrm{~d}$ ).

When the robotic fish was in the white half of the test tank the time spent by fish in the white half was significantly longer than the time spent in the black half $(Z=5.261 ; P$ $<0.0001$ ) (Fig. 5a).

When the robotic fish was in the black half of the test tank the time spent by fish in the black half was significantly longer than the time spent in the white half $(Z=-5.396 ; P$ $<0.0001)$ (Fig. 5b).

When the robotic fish was not present the test tank the time spent by fish in the black half was significantly longer than the time spent in the white half $(Z=-5.396 ; P<0.0001)$ (Fig. 5c).

\section{Discussion}

In this study on animal-robot interaction, we show how social robots can contribute to facilitate current research on anxiety, and provide the evidence that can be potentially exploited to ameliorate anxiety-related disorders. Anxiety is a major psychiatric issue whose aetiology is associated with the interaction of several psychosocial factors which lead to neurobiological and neuropsychological dysfunctions [44]. Social robots, used in several healthcare contexts, can represent a strategic method to treat anxiety [45].

In the first experiment we tested beneficial effects resulting from the presence of a conspecific-mimicking robotic fish when a fish individual was transferred in a new tank. It is well established that a new environment causes a stress in fish whose the indicating-anxiety response is represented by the tendency to locate in the bottom of the tank [38, 46, 47]. However, when the robotic fish was present in the test tank, $P$. innesi individuals spent a significantly longer time in the upper section of the test tank, clearly indicating a reduction of their state of anxiety due to social stimuli. Also latency lasted considerably less when the robotic fish was exposed, likely do to the effect of social stimuli from the robotic fish that elicited quicker normal foraging activities [48]. These evidences can be associated with a phenomenon widespread in humans and other animals named social buffering [49]. Such a phenomenon, regulated by specific neural mechanisms [50-52], consists in a better recovery from an aversive event due to social support. Results from the second experiment further confirmed this hypothesis. P. innesi, 


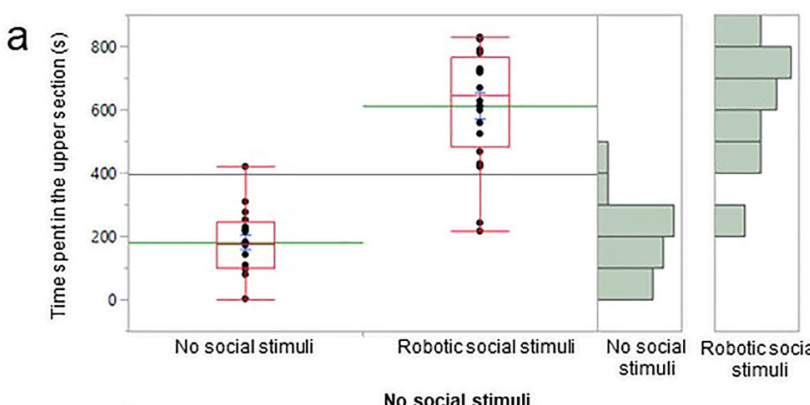

b

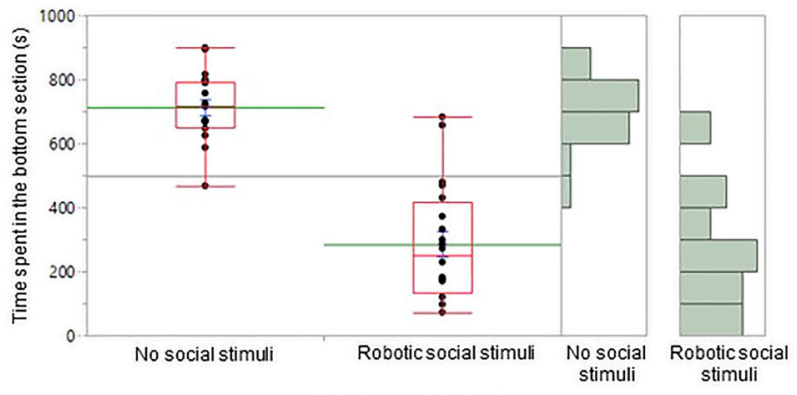

C

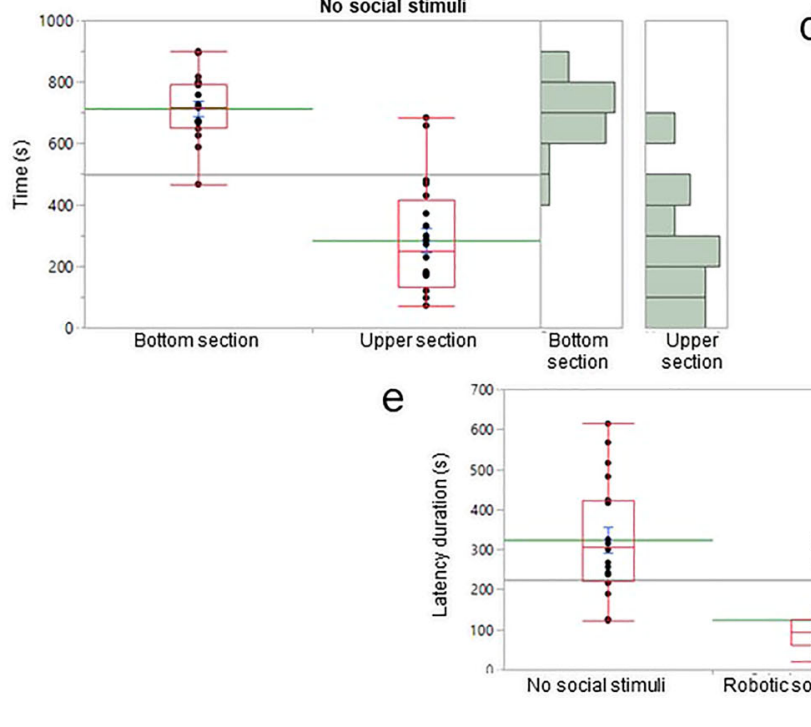

d

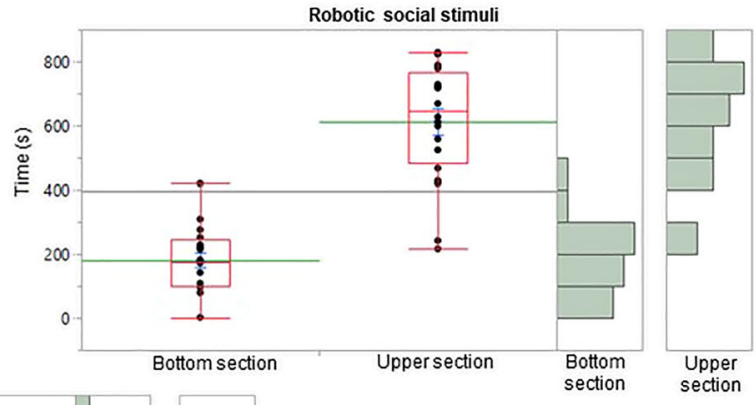

Fig. 3 Paracheirodon innesi anxiety-related responses post-exposure to the robotic fish in the experiment 1 , including a the time spent in the upper section of the test tank, and $\mathbf{b}$ the time spent in bottom section of the test tank, when the robotic fish was presented, and where no social stimuli were presented in the test tank; $\mathbf{c}$ the effect of no social stimuli, and $\mathbf{d}$ of the robotic fish on the time spent by fish in the two section of the test tank; e latency duration in $P$. innesi post-exposure to the robotic fish and to no social stimuli. In the box plots are included the median (red line) and their lower and upper quartiles and outliers, and green lines and blue T-bars showing mean and standard error values, respectively. On the right of each box plot, histograms showing data distribution are reported together with other teleost species, is a scototactic fish, preferring dark environments [24, 39]. Nevertheless, when the robotic fish was placed in the white half of the test tank, real neon tetras preferentially swam in this section otherwise aversive in different treatments. Furthermore, the time needed for the first choice of half was significantly shorter in presence of the robotic fish in one of the two halves of the test tank than in absence of social stimuli. Also, the schooling behaviour duration was not importantly affected by the location of the robotic fish. In general, fish may be involved in novel object investigation displays when exposed to a new stimulus, and several studies addressed this issue to understand how to manipulate important parameters for social affiliation of fish with robotic agents, including the speed and the trajectory of the artifact, as well as its shape, colour, and flexibility [53-55]. However, it should be noted that in the experimental conditions of this study (e.g. isolating an individual of a social species and keep it in a new environment) the novel object investigation behaviour would be strongly inhibited, and neophobia, reduced exploration, or hesitancy would predominate [56, 57]. Conversely, in our case the robotic fish had a very clear effect in reducing the anxiety behaviour of fish in these adverse conditions, supporting the idea that the artifact triggered social attraction in animals due to its recognizable biomimetic features, as also reported in previous studies on different taxa interacting with conspecific-mimicking robots $[58,59]$. Thus, we suggest that the effectiveness of robotic social stimuli in buffering anxiety is of particular relevance, since the robotic fish directly influenced fish individual behaviours by increasing their boldness thanks to a conspecific-like presence.

Our findings on neon tetras are valuable to understand the role of social robots in modulating neural mechanisms of social buffering and anxiety control also in humans, since teleosts present brain regions that are homologous to those of mammals [60-63]. Few studies in mammals have shown that a decrease of the anxiety status occurring in the presence of a conspecific is associated to a lower activation of 
a

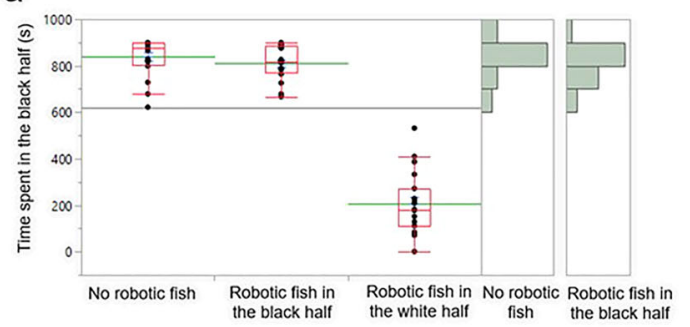

C

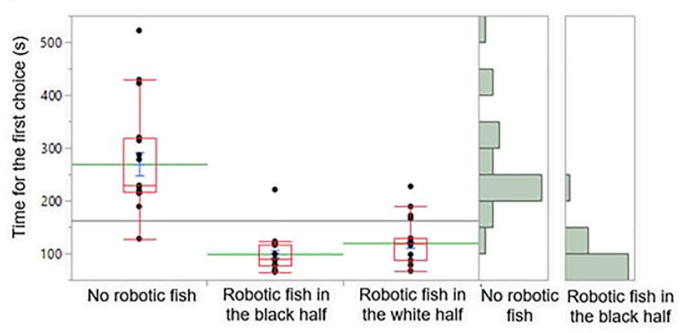

b
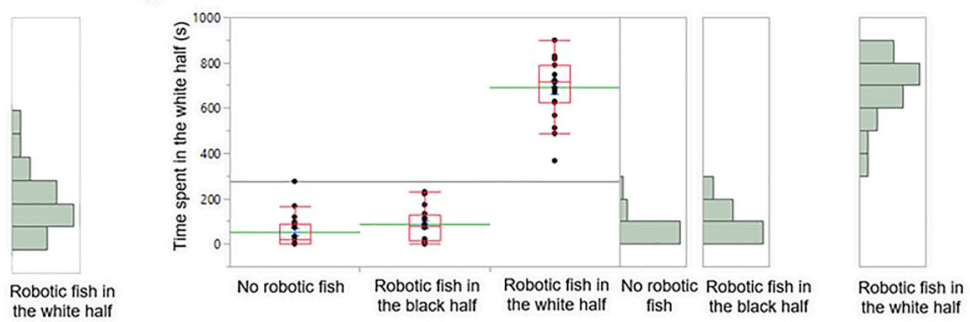

d

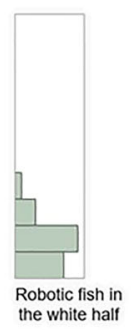

Fig. 4 Paracheirodon innesi anxiety-related responses post-exposure to the robotic fish in the experiment 2, including a the time spent in the black half of the test thank, $\mathbf{b}$ the time spent in the white half of the test thank, $\mathbf{c}$ the time needed for the first choice, and $\mathbf{d}$ the schooling behaviour duration. In the box plots are included the median (red line)

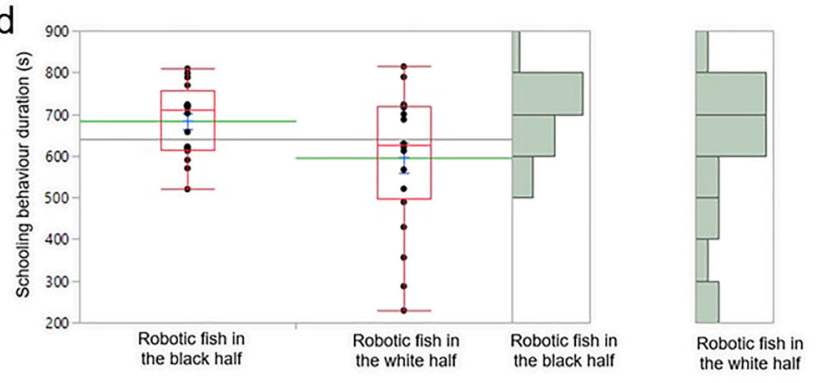

and their lower and upper quartiles and outliers, and green lines and blue T-bars showing mean and standard error values, respectively. On the right of each box plot, histograms showing data distribution are reported

b Robotic fish in the black half
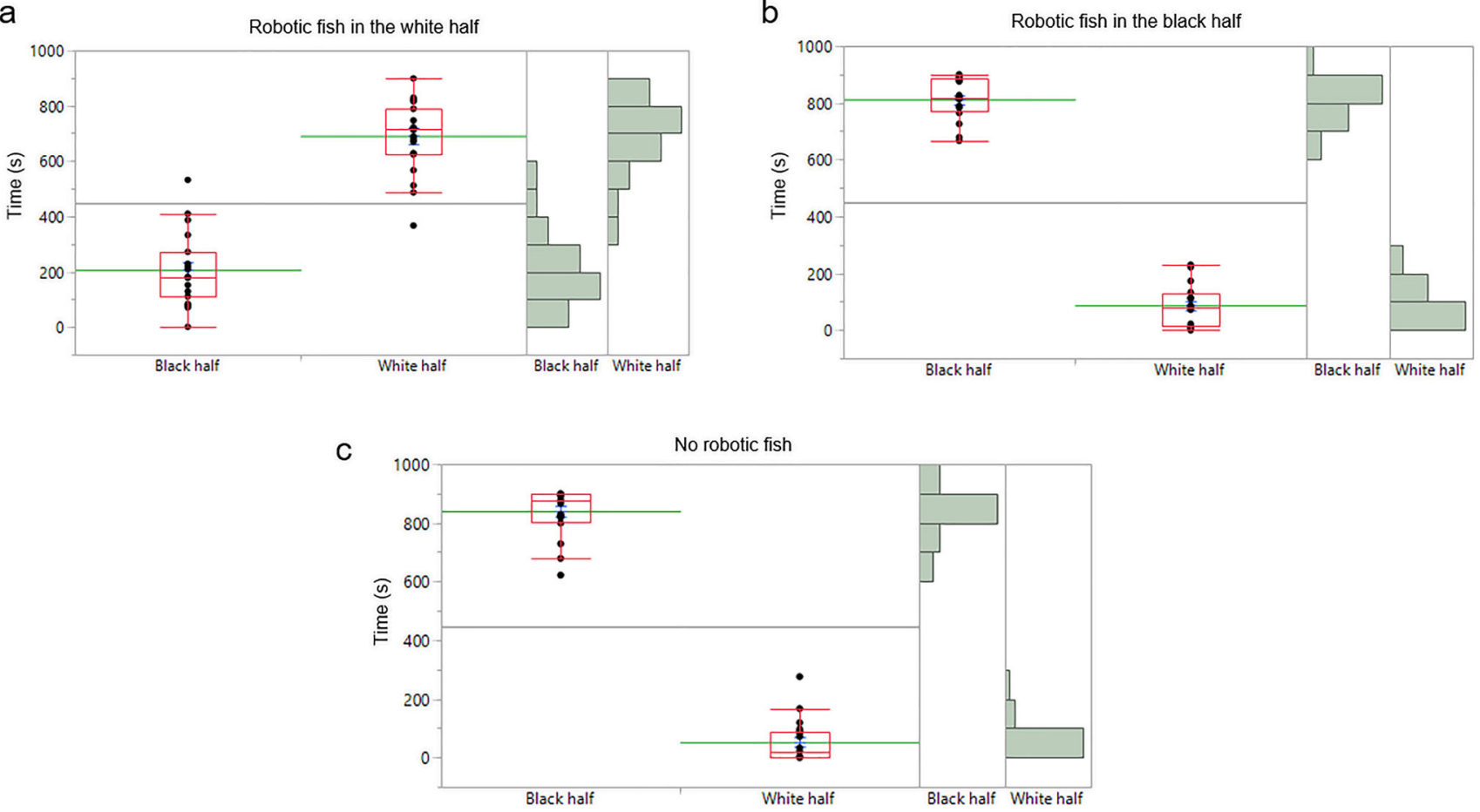

Fig. 5 Paracheirodon innesi anxiety-related responses post-exposure to the robotic fish in the experiment 2 showing a the effect of the robotic fish in white half of the test tank, $\mathbf{b}$ the effect of the robotic fish in black half of the test tank, and $\mathbf{c}$ the effect of no robotic fish in the test tank, on the time spent in the black half and in the white half of the test tank.
In the box plots are included the median (red line) and their lower and upper quartiles and outliers, and green lines and blue T-bars showing mean and standard error values, respectively. On the right of each box plot, histograms showing data distribution are reported 
the hypothalamus paraventricular nucleus, as well as the lateral and the central amygdala [52, 64, 65]. Concerning teleost fish, Faustino et al. [49] accurately dissected the neural mechanisms of anxiety reduction resulting from social stimuli in zebrafish, showing that social buffering produced a specific co-activation pattern, involving the medial zone of the dorsal telencephalic area, the supracommissural nucleus of the ventral telencephalic area, and the preoptic area, that are supposed to be homologues of mammals' brain nuclei [63].

This evidence suggests a remarkable evolutionary stable mechanism in controlling anxiety that can be pragmatically crucial for social robotics engineering design, and for robotic-based anxiety treatments. In future research, advanced robot architectures will be implemented (i.e. the Sense-Act-Modulated-by-Interactions architecture [66]), to further increase the interactive features of the experimental apparatus.

This pioneer study offers basic information to unveil the evolution and mechanisms of social buffering to regulate anxiety in social species. Furthermore, it highlights the key role of life-like robotic agents in attenuating anxiety symptoms in clinical contexts, as well as in animal healthcare and welfare.

\section{Conclusion}

The present pilot study provides the evidence that animalrobot interactions can be used to advance current research on anxiety, as well as to investigate the role of social robots as a therapy to ameliorate anxiety disorders. Although humas are aware that robots are not conspecifics, unlike our fish experiment, social robotics often identifies, extracts and models principles of human-human interaction to mirror them in robots, using human communication channels, emotion expression, etc., to interact with humans. So, this research on animal-robot interaction provide useful insights to reflect this process in humans. Social support provided by the robotic fish mimicking a $P$. innesi conspecific produced a better recovery of normal behaviours in real $P$. innesi individuals when in aversive contexts producing anxiety responses. This phenomenon, named social buffering, is regulated by specific neural mechanisms, and social robots can be used to modulate them to control anxiety.

These findings open a new research avenue for investigating the evolution and mechanisms of social buffering to reduce anxiety, as well as for using social robots as an alternative to traditional approaches to treat anxiety symptoms.

Funding Open access funding provided by Scuola Superiore Sant'Anna within the CRUI-CARE Agreement. Financial support was received from the H2020 FETOPEN Project "Robocoenosis-ROBOts in cooperation with a bioCOENOSIS" [899520]. Funders had no role in the study design, data collection and analysis, decision to publish, or preparation of the manuscript.

\section{Declarations}

Conflict of interest The authors declare that they have no conflict of interest.

Ethical approval This study was carried out in accordance with the Guidelines for the Use of Animals in Research, and the 7010-2020-IEEE Recommended Practice for Assessing the Impact of Autonomous and Intelligent Systems on Human Well-Being, as well as the legal requirements of Italian and EU legislation. All experiments consisted in behavioural tests, and no specific permits are needed in the country where the experiments were conducted.

Open Access This article is licensed under a Creative Commons Attribution 4.0 International License, which permits use, sharing, adaptation, distribution and reproduction in any medium or format, as long as you give appropriate credit to the original author(s) and the source, provide a link to the Creative Commons licence, and indicate if changes were made. The images or other third party material in this article are included in the article's Creative Commons licence, unless indicated otherwise in a credit line to the material. If material is not included in the article's Creative Commons licence and your intended use is not permitted by statutory regulation or exceeds the permitted use, you will need to obtain permission directly from the copyright holder. To view a copy of this licence, visit http://creativecomm ons.org/licenses/by/4.0/.

\section{References}

1. Fong T, Nourbakhsh I, Dautenhahn K (2003) A survey of socially interactive robots. Robot Auton Syst 42(3-4):143-166

2. Dautenhahn K (2007) Socially intelligent robots: dimensions of human-robot interaction. Philos Trans R Soc B: Biol Sci 362(1480):679-704

3. Yan H, Ang MH, Poo AN (2014) A survey on perception methods for human-robot interaction in social robots. Int J Soc Robot $6(1): 85-119$

4. Breazeal C, Dautenhahn K, Kanda T (2016) Social robotics. In: Siciliano B, Khatib O (eds) Springer handbook of robotics. Springer, Cham, pp 1935-1972

5. Matarić MJ (2017) Socially assistive robotics: Human augmentation versus automation. Sci Robot 2(4):eaam 5410

6. Breazeal C, Scassellati B (1999, October) How to build robots that make friends and influence people. In Proceedings 1999 IEEE/RSJ International Conference on Intelligent Robots and Systems. Human and Environment Friendly Robots with High Intelligence and Emotional Quotients (Cat. No. 99CH36289) (Vol. 2, pp 858-863). IEEE.

7. Fink $\mathbf{J}$ (2012, October) Anthropomorphism and human likeness in the design of robots and human-robot interaction. In International Conference on Social Robotics (pp 199-208). Springer, Berlin, Heidelberg.

8. Mann JA, MacDonald BA, Kuo IH, Li X, Broadbent E (2015) People respond better to robots than computer tablets delivering healthcare instructions. Comput Hum Behav 43(C):112-117

9. Jeong S, Logan D E, Goodwin M S, Graca S et al (2015, March) A social robot to mitigate stress, anxiety, and pain in hospital pediatric care. In Proceedings of the Tenth Annual ACM/IEEE International Conference on Human-Robot Interaction Extended Abstracts (pp 103-104).

10. Bernstein GA, Borchardt CM, Perwien AR (1996) Anxiety disorders in children and adolescents: A review of the past 10 years. $\mathrm{J}$ Am Acad Child Adolesc Psychiatr 35(9):1110-1119 
11. Bar-Haim Y (2010) Research review: attention bias modification (ABM): a novel treatment for anxiety disorders. J Child Psychol Psychiatr 51(8):859-870

12. Kessler RC, Chiu WT, Demler O, Walters EE (2005) Prevalence, severity, and comorbidity of 12-month DSM-IV disorders in the National Comorbidity Survey Replication. Arch Gen Psychiatr 62(6):617-627

13. Cattell RB (1966) Anxiety and motivation: Theory and crucial experiments. Anxiety and Behav 1:23-62

14. Rossi S, Larafa M, Ruocco M (2020) Emotional and behavioural distraction by a social robot for children anxiety reduction during vaccination. Int J Soc Robot. https://doi.org/10.1007/s12369-01900616-w

15. van Straten CL, Peter J, Kühne R (2020) Child-robot relationship formation: A narrative review of empirical research. Int J Soc Robot 12(2):325-344

16. Blanchard RJ, Yudko EB, Rodgers RJ, Blanchard DC (1993) Defense system psychopharmacology: an ethological approach to the pharmacology of fear and anxiety. Behav Brain Res 58(1-2):155-165

17. Rodgers RJ, Cao BJ, Dalvi A, Holmes A (1997) Animal models of anxiety: an ethological perspective. Braz J Med Biol Res 30:289-304

18. Kalueff AV, Wheaton M, Murphy DL (2007) What's wrong with my mouse model?: Advances and strategies in animal modeling of anxiety and depression. Behav Brain Res 179(1):1-18

19. Maximino C, Marques T, Dias F, Cortes F V et al (2007) A comparative analysis of the preference for dark environments in five teleosts. Int J Comp Psychol 20(4)

20. Maximino C, de Oliveira DL, Rosemberg DB, Batista EDJO et al (2012) A comparison of the light/dark and novel tank tests in zebrafish. Behav 149(10-12):1099-1123

21. Bracke MBM, Metz JHM, Dijkhuizen AA, Spruijt BM (2001) Development of a decision support system for assessing farm animal welfare in relation to husbandry systems: strategy and prototype. J Agric Environ Ethics 14(3):321-337

22. Noordhuizen JPTM, Metz HM (2005) Quality control on dairy farms with emphasis on public health, food safety, animal health and welfare. Stočarstvo: Časopis za unapređenje stočarstva 59(1):39-55

23. Cooke M (2016) Animal welfare in farmed fish. Bus Benchmark Farm Animal Welf, Invest Brief 23:1-16

24. Maximino C, De Brito TM, de Mattos DCAG, Gouveia A Jr, Morato S (2010) Scototaxis as anxiety-like behavior in fish. Nat Protoc 5(2):209

25. Helfman G, Collette BB, Facey DE, Bowen BW (2009) The diversity of fishes: biology, evolution, and ecology. Wiley, New York

26. Stewart A, Wu N, Cachat J, Hart P et al (2011) Pharmacological modulation of anxiety-like phenotypes in adult zebrafish behavioral models. Prog Neuropsychopharmacol Biol Psychiatry 35(6):1421-1431

27. Geisler R (1979) Exploring the habitat of the neon tetra. Aquar Dig Int 24:24-27

28. Chapman FA, Colle DE, Rottmann RW, Shireman JV (1998) Controlled spawning of the neon tetra. Progr Fish-Cultur 60(1):32-37

29. Krause J, Winfield AF, Deneubourg JL (2011) Interactive robots in experimental biology. Trends Ecol Evol 26(7):369-375

30. Polverino G, Karakaya M, Spinello C, Soman VR, Porfiri M (2019) Behavioural and life-history responses of mosquitofish to biologically inspired and interactive robotic predators. J R Soc Interface 16(158):20190359

31. Romano D, Donati E, Benelli G, Stefanini C (2019) A review on animal-robot interaction: from bio-hybrid organisms to mixed societies. Biol Cybern 113(3):201-225
32. Romano D, Benelli G, Stefanini C (2019b) Encoding lateralization of jump kinematics and eye use in a locust via bio-robotic artifacts. J Exp Biol 222(2)

33. Romano D, Elayan H, Benelli G, Stefanini C (2020) Together we stand-analyzing schooling behavior in naive newborn guppies through biorobotic predators. J Bionic Eng 17(1):174-184

34. Romano D, Benelli G, Kavallieratos NG, Athanassiou CG, Canale A, Stefanini C (2020) Beetle-robot hybrid interaction: sex, lateralization and mating experience modulate behavioural responses to robotic cues in the larger grain borer Prostephanus truncatus (Horn). Biol Cybern 114(4):473-483

35. Romano D, Bloemberg J, Tannous M, Stefanini C (2020) Impact of aging and cognitive mechanisms on high-speed motor activation patterns: evidence from an orthoptera-robot interaction. IEEE Trans Med Robot Bionics 2(2):292-296

36. Datteri E (2020) The logic of interactive biorobotics. Front Bioeng Biotechnol 8:637. https://doi.org/10.3389/fbioe.2020.00637

37. Clément RJ, Macrì S, Porfiri M (2020) Design and development of a robotic predator as a stimulus in conditioned place aversion for the study of the effect of ethanol and citalopram in zebrafish. Behav Brain Res 378:112256

38. Levin ED, Bencan Z, Cerutti DT (2007) Anxiolytic effects of nicotine in zebrafish. Physiol Behav 90(1):54-58

39. Hope BV, Hamilton TJ, Hurd PL (2019) Submerged plus maze: A novel test for studying anxiety-like behaviour in fish. Behav Brain Res 362:332-337

40. ASAB, ABS, (2014) Guidelines for the treatment of animals in behavioural research and teaching. Anim Behav 87:1-9

41. Olszewska J I (2020) IEEE Recommended practice for assessing the impact of autonomous and intelligent systems on human wellbeing: IEEE Stand 7010-2020.

42. European Commission (2007) Commission recommendations of 18 June 2007 on guidelines for the accommodation and care of animals used for experimental and other scientific purposes. Annex II to European Council Directive 86/609. See 2007/526/ EC. Retrieved from http://eurex.europa.eu/LexUriServ/LexUriServ. do? uri1/4OJ:L. 2007.197:0001:0089:EN:PDF.

43. Seghers BH, Magurran AE (1994) Predator inspection behaviour covaries with schooling tendency amongst wild guppy, Poecilia reticulata, populations in Trinidad. Behav 128(1-2):121-134

44. Bandelow B, Michaelis S, Wedekind D (2017) Treatment of anxiety disorders. Dialogues Clin Neurosci 19(2):93

45. Dawe J, Sutherland C, Barco A, Broadbent E (2019) Can social robots help children in healthcare contexts? A scoping review. BMJ Paediatr Open 3(1)

46. Tran ST, Gerlai R (2016) The novel tank test: handling stress and the context specific psychopharmacology of anxiety. Current Psychopharmacol 5(2):169-179

47. Kysil EV, Meshalkina DA, Frick EE, Echevarria DJ et al (2017) Comparative analyses of zebrafish anxiety-like behavior using conflict-based novelty tests. Zebrafish 14(3):197-208

48. Vadas RS, Burrows MT, Hughes RN (1994) Foraging strategies of dogwhelks, Nucella lapillus (L): interacting effects of age, diet and chemical cues to the threat of predation. Oecologia 100(4):439-450

49. Faustino AI, Tacão-Monteiro A, Oliveira RF (2017) Mechanisms of social buffering of fear in zebrafish. Sci Rep 7:44329

50. Kiyokawa Y, Takeuchi Y, Mori Y (2007) Two types of social buffering differentially mitigate conditioned fear responses. Eur J Neurosci 26(12):3606-3613

51. Smith AS, Wang Z (2014) Hypothalamic oxytocin mediates social buffering of the stress response. Biol Psychiat 76(4):281-288

52. Fuzzo F, Matsumoto J, Kiyokawa Y, Takeuchi Y, Ono T, Nishijo H (2015) Social buffering suppresses fear-associated activation of the lateral amygdala in male rats: behavioral and neurophysiological evidence. Front Neurosci 9:99 
53. Bonnet F, Kato Y, Halloy J, Mondada F (2016) Infiltrating the zebrafish swarm: design, implementation and experimental tests of a miniature robotic fish lure for fish-robot interaction studies. Artif Life Robot 21(3):239-246

54. Landgraf T, Bierbach D, Nguyen H, Muggelberg N, Romanczuk P, Krause J (2016) RoboFish: increased acceptance of interactive robotic fish with realistic eyes and natural motion patterns by live Trinidadian guppies. Bioinspir Biomime 11(1):015001

55. Larsch J, Baier H (2018) Biological motion as an innate perceptual mechanism driving social affiliation. Curr Biol 28(22):3523-3532

56. Thomas RJ, King TA, Forshaw HE, Marples NM, Speed MP, Cable J (2010) The response of fish to novel prey: evidence that dietary conservatism is not restricted to birds. Behav Ecol 21(4):669-675

57. Martins CI, Silva PI, Conceição LE, Costas B, Höglund E, Øverli $\varnothing$, Schrama JW (2011) Linking fearfulness and coping styles in fish. PLoS ONE 6(11):e28084

58. Romano D, Benelli G, Stefanini C (2021) Opposite valence social information provided by bio-robotic demonstrators shapes selection processes in the green bottle fly. J R Soc Interface 18(176):20210056. https://doi.org/10.1098/rsif.2021.0056

59. Romano D, Stefanini C (2021) Unveiling social distancing mechanisms via a fish-robot hybrid interaction. Biol Cybern. https://doi. org/10.1007/s00422-021-00867-9

60. O'Connell LA, Hofmann HA (2011) The vertebrate mesolimbic reward system and social behavior network: a comparative synthesis. J Comp Neurol 519(18):3599-3639

61. Ganz J, Kaslin J, Freudenreich D, Machate A, Geffarth M, Brand M (2012) Subdivisions of the adult zebrafish subpallium by molecular marker analysis. J Comp Neurol 520(3):633-655

62. Maximino C, Lima MG, Oliveira KRM, Batista EDJO, Herculano AM (2013) "Limbic associative" and "autonomic" amygdala in teleosts: a review of the evidence. J Chem Neuroanat 48:1-13

63. Teles MC, Cardoso SD, Oliveira RF (2016) Social plasticity relies on different neuroplasticity mechanisms across the brain social decision-making network in zebrafish. Front Behav Neurosci 10:16. https://doi.org/10.3389/fnbeh.2016.00016

64. Da Costa AP, Leigh AE, Man MS, Kendrick KM (2004) Face pictures reduce behavioural, autonomic, endocrine and neural indices of stress and fear in sheep. Proc Royal Soc Lond Ser B: Biol Sci 271(1552):2077-2084

65. Takahashi Y, Kiyokawa Y, Kodama Y, Arata S, Takeuchi Y, Mori Y (2013) Olfactory signals mediate social buffering of conditioned fear responses in male rats. Behav Brain Res 240:46-51
66. Calzado J, Lindsay A, Chen C, Samuels G, Olszewska J I (2018). SAMI: interactive, multi-sense robot architecture. In 2018 IEEE 22nd International Conference on Intelligent Engineering Systems (INES) (pp 000317-000322). IEEE.

Publisher's Note Springer Nature remains neutral with regard to jurisdictional claims in published maps and institutional affiliations.

Donato Romano [M.Sc. in Agriculture Science and Technologies (honors), $\mathrm{PhD}$ in BioRobotics (honors)] is currently Assistant Professor at The BioRobotics Institute of Scuola Superiore Sant'Anna of Pisa, Italy, and teaches course in the M.S. Program in Bionics Engineering jointly organized by the University of Pisa and by the Scuola Superiore Sant'Anna. Romano is mainly focusing his activities on bioinspired and biomimetic robotics, and in particular on animal-robot interaction, biohybrid systems, natural and biohybrid intelligence, ethorobotics, neuroethology. He received national and international recognitions for his research. He also has been visiting researcher at Khalifa University, Abu Dhabi (UAE). He is Member of the Editorial Board for many International Scientific Journals.

Cesare Stefanini [M.Sc. in Mech. Eng. (honors), PhD in Microengineering (honors)] is Full Professor of Bioengineering at the BioRobotics Institute of Scuola Superiore Sant'Anna of Pisa, Italy, with the role of Area Leader in "Creative Engineering Design". His research activity is applied to different fields, including biomechatronics, and micromechatronics for medical and industrial applications. He received international recognitions for the development of novel actuators for microrobots and he has been visiting researcher at the University of Stanford, Center for Design Research, and Professor at Khalifa University, UAE. 